\title{
STARCOUNTS IN THE HUBBLE DEEP FIELD:
}

\section{FEWER THAN EXPECTED, MORE THAN EXPECTED}

The Halo main-sequence $\mathcal{E}$ Halo white-dwarf luminosity functions

\author{
R. A. MÉNDEZ ${ }^{1}$, G. DE MARCHI ${ }^{1}$, D. MINNITI ${ }^{2}$, \\ A. BAKER ${ }^{3}$ AND W. J. COUCH ${ }^{4}$ \\ ${ }^{1}$ European Southern Observatory \\ ${ }^{2}$ Lawrence Livermore National Laboratory \\ ${ }^{3}$ Institute of Astronomy \\ ${ }^{4}$ School of Physics, University of New South Wales
}

\section{Introduction: The Hubble Deep Field}

The Hubble Deep Field (HDF, Williams et al. 1996), recently acquired with the Hubble Space Telescope, is the deepest, most detailed optical view of the Universe. Even though intended primarily for the study of the high-redshift Universe, the HDF provides a unique opportunity to find faint stellar objects in our Galaxy, set constraints on number of low-mass Halo stars, estimate the contribution of baryonic matter to the dark Halo, investigate the nature of microlensing sources, and to calibrate Galactic Structure models. The HDF has been an STScI initiative to provide the deepest exposures yet acquired with HST on a non-proprietary basis. Observations of an 'anonymous field' located at $l=125.9^{\circ}, b=54.8^{\circ}$ were performed on the continuous viewing zone, for a total of 150 orbits (10 consecutive days) in the four HST passbands F300W, F450W, F606W and F814W. These observations reached $5 \sigma$ magnitude limits (for Galaxies) of roughly (STMAG) $30^{\text {th }}$ mag in all these passbands (except F300W which reaches $27^{\text {th }} \mathrm{mag}$ ). We have used a novel software detection \& classification algorithm to create a sample of point-like objects in the HDF. We have also compared the observed stellar counts to constrain the faint end of the Halo field luminosity function by using a recent Galactic Structure model. The unexpected appearance of a faint-blue group of very compact objects is discussed as well. 


\section{Analysis of the Hubble Deep Field: A Tale of a Few}

As a result of combining groups of exposures with slight telescope offsets, the final Wide-Field camera images have a resolution of 0.04 arcsec pixel $^{-1}$ and, therefore, stellar images are actually subsampled (Williams et al. 1996). In our strategy, the F606W and F814W images were coadded for detections at the faintest levels allowed by these data, totalling $70 \mathrm{~h}$ of combined exposure time. The field of view is $4.69 \mathrm{arcmin}^{2}$.

To create our point-like sample we have used a recently developed source extraction algorithm (SExtractor: Bertin \& Arnouts 1996). This software uses a neural network classifier, trained with observed and simulated stars and galaxies. In addition to providing astrometry and photometry, the software also gives a 'Stellarity Index' (or CLASS). The CLASS parameter can be viewed as the probability of an object being a star; it goes from 0 for extended objects to 1 for point-like objects. We have found that objects with $C L A S S<0.85$ are clearly extended on the frames. On this basis, we have compiled a sample of 16 objects satisfying the criteria that their $C L A S S \geq 0.85$, these objects do not seem to exhibit any structure around them.

We are quite confident that we are not missing stars down to $F 606 \mathrm{~W} \sim$ $F 814 W \sim 30 \mathrm{mag}$, while the inclusion of some compact objects could be a problem for magnitudes fainter than $F 814 W \sim 27.5$. More details about the catalogue creation and completeness can be found in Méndez et al. (1996)

\section{Model Comparison and Conclusions}

A detailed comparison of the observed counts on the HDF and the counts prediced by a recent model by Méndez and van Altena (1996) is given in Méndez et al. (1996). The main result is that the luminosity function for faint $\left(12 \leq M_{V} \leq 14\right)$ Halo stars is depressed by, at least, a factor of two (but most likely a factor of four) with respect to the luminosity function for nearby stars by Wielen et al. (1983). These results are coincident with recent findings from deep HST observations of Globular Clusters (De Marchi \& Paresce 1995, and references therein) that indicate a sharp decrease in stars fainter than $M_{v} \sim 12$. Also, recent determinations of the disk luminosity functions from HST observations indicate that the faint end of the Wielen et al. (1983) luminosity function is indeed overpopulated, and that the disk luminosity function also has a maximum at $M_{v} \sim 12$ and a steep decrease at fainter magnitudes (Santiago et al. 1996). This would seem to indicate that the luminosity function of the different stellar populations in our Galaxy behave in a similar way, and that they are not strongly dependent on metallicity. 
A group of faint-blue objects (with $25 \leq V \leq 28,-0.5<B-V<0.5$ and $0<V-I<1.2$ ) is found to be too blue at these magnitudes to be QSOs. If they are assumed to be stellar objects they would be Halo whitedwarfs (WDs). Their numbers would indicate an extreme overdensity of Halo WDs with respect to theoretical expectations. The mass locked in these WDs would be on the order of $10^{-4} M_{\odot} p c^{-3}$, and their local number density would be as high as $40 \%$ as that of disk WDs. An alternative to scaling the Halo WD luminosity function would be to assume a sharp increase in the number of Halo WDs for $M_{v} \geq 11$. The colors of these putative WDs roughly agree with the theoretical colors computed by Bergeron et al. (1995), indicating temperatures in the range of $T_{\text {eff }} \sim 7-8 \times 10^{3} \mathrm{~K}$. Based on luminosities derived from distances computed from the Galactic models (roughly 8 to $19 \mathrm{kpc}$ ), we estimate cooling ages of less than $1 \mathrm{Gyr}$, similar to the WDs found by Richer et al. (1995) in the globular cluster M4.

The faint-blue objects lay at the boundary of our star-galaxy boundary $(C L A S S=0.85)$ and therefore we can not rule out the (more likely) possibility that this objects are distant star-forming regions, perhaps globular clusters or dwarf ellipticals in the process of forming, as discussed by Elson et al. (1996).

\section{References}

Bergeron, P., Wesemael, F., Beauchamp, A., 1995, Publ.Astron.Soc.Pacific, 107, 1047

Bertin, E., and Arnouts, S., 1996, Astron.Astrophys.Suppl., 117, 393

De Marchi, G., \& Paresce, F. 1995, Astron.Astrophys., 304, 211

Elson, R.A.W., Santiago, B.X., and Gilmore, G.F., 1996, New Astronomy, in press

Méndez, R.A. and van Altena, W.F., 1996, Astron.J., 112, 655

Méndez, R.A., Minniti, D., De Marchi, G., Baker, A., Couch, W.J., 1996, Mon.Not.R.astron.Soc., in press

Richer, H.B., Fahlmann, G.G., Ibata, R.A., Stetson, P.B., Bell, R.A., Bolte, M., Bond, H.E., Harris, W.E., Hesser, J.E., Mandushev, G., Pryor, C., and VandenBerg, D.A., 1995, Astrophys.J., 451, L17

Santiago, B.X., Gilmore, G., and Elson, R.A.W., 1996, Mon.Not.R.astron.Soc., 281, 871

Wielen, R., Jahrei $\beta$, H., Krüger, R. , 1983, in The Nearby Stars and the Stellar Luminosity Function, IAU Colloq. No. 76, eds. A.G. Davis Philip and A.R. Upgren (L. Davis Press, Schenectady), p. 163

Williams, R.E., et al., 1996, Astron.J., in press 